\title{
ON THE DOUBLE COMMUTANT EXPECTATION PROPERTY FOR OPERATOR SYSTEMS
}

\begin{abstract}
FLORIN POP
Abstract. In this note we present an alternative viewpoint to the Double Commutant Expectation Property (DCEP) for operator systems, introduced by Kavruk, Paulsen, Todorov and Tomforde. Our approach is based on the universal $C^{*}$-algebra of an operator system and is used to obtain new proofs of several results for operator systems analogue to the properties $C^{*}$-algebras have. Mathematics subject classification (2010): 46L06, 46L07.

Keywords and phrases: Operator system, weak expectation.
\end{abstract}

\section{REFERENCES}

[1] W. B. ARVeson, Notes on extensions of $C^{*}$-algebras, Duke Math. J. 44 (1977), 329-355.

[2] N. P. BRown, N. OzAWA, $C^{*}$-algebras and finite-dimensional approximations, Graduate Studies in Mathematics, 88, American Mathematical Society, Providence, RI, 2008.

[3] M.-D. Choi And E. G. EfFros, The completely positive lifting problem for $C^{*}$-algebras, Ann. Math. 104 (1976), 585-609.

[4] U. HaAgerup, G. Pisier, Linear operators between $C^{*}$-algebras, Duke Math. J. 71 (1993), 889925.

[5] K. H. HAN, On maximal tensor products and quotient maps of operator systems, J. Math. Anal. Appl. 384 (2011), 375-386.

[6] T. HuRUYA, On compact completely bounded maps of $C^{*}$-algebras, Michigan Math. J. 30 (1983), 213-220.

[7] A. Kavruk, V. I. Paulsen, I. Todorov, M. Tomforde, Tensor products of operator systems, J. Funct. Anal. 261 (2011), 267-299.

[8] A. Kavruk, V. I. Paulsen, I. Todorov, M. Tomforde, Quotients, exactness and nuclearity in the operator system category, Adv. Math. 235 (2013), 321-360.

[9] E. KIRCHBERG, On nonsemisplit extensions, tensor products and exactness of group $C^{*}$-algebras, Invent. Math. 112 (1993), 449-489.

[10] E. KIRCHBERG, Commutants of unitaries in UHF algebras and functorial properties of exactness, J. Reine Angew. Math. 452 (1994), 39-77.

[11] E. Kirchberg, S. WASSERmann, $C^{*}$-algebras generated by operator systems, J. Funct. Anal. 155 (1998), 324-351.

[12] E. C. LANCE, On nuclear $C^{*}$-algebras, J. Funct. Anal. 12 (1973), 157-176.

[13] N. OZAWA, About the QWEP conjecture, Int. J. Math. 15 (2004), 501-530.

[14] V. I. PAUlSEN, Completely biunded maps on $C^{*}$-algebras and invariant operator ranges, Proc. Amer. Math. Soc. 86 (1982), 91-96.

[15] V. I. PAULSEN, Every completely polynomially bounded operator is similar to a contraction, J. Funct. Anal. 55 (1984), 1-17.

[16] V. I. Paulsen, Completely Bounded Maps and Operator Algebras, Cambridge University Press, 2003.

[17] G. PISIER, A simple proof of a theorem of Kirchberg and related results on $C^{*}$-norms, J. Operator Theory 35 (1996), 317-335.

[18] G. PISIER, Introduction to Operator Space Theory, Cambridge University Press, 2003. 